Andrews, M and Gatersleben, B (2010). Variations in perceptions of danger, fear and preference in a simulated natural environment, Journal of Environmental Psychology, 30, 473-481

\title{
Variations in perceptions of danger, fear and preference in a simulated natural environment
}

Andrews, Matt and Gatersleben, Birgitta

\section{Abstract}

Although natural environments can help promote health, they also contain a number of dangers. This study attempted to examine how variations in the physical structure of a simulated natural environment influenced perceptions of both overall and specific types of danger, fear and preference before exploring the relationships between these variables. Three simulated walks through a natural environment differing in levels of prospectrefuge according to Fisher and Nasar's (1992) typology of prospect-refuge were created for the study. Respondents were randomly assigned to one of the conditions and asked to imagine taking the walk for real. In support of the typology, the results found that the walks with higher levels of prospect-refuge (higher visibility, fewer hiding places and more accessibility) were perceived as less dangerous and fearful and more preferred than walks with lower levels of prospect-refuge. However despite levels of prospect-refuge appearing to impact on the perceived likelihood of encountering a physical danger or becoming lost, they were not found to impact on the perception of encountering a social danger. 
Keywords: Prospect-refuge; preference; nature; perceived danger; fear

\section{Introduction}

Intuition would lead us to believe that people are more likely to prefer an environment and engage with it if they perceive it as being a safe environment. A consistent negative relationship has emerged between perceived danger and preference in urban environments (e.g. Blöbaum \& Hunecke, 2005; Fisher \& Nasar, 1992; Herzog \& Flynn-Smith, 2001; Herzog \& Miller, 1988; Nasar \& Fisher, 1993). One key factor that may influence perceptions of safety and danger is the physical structure of an environment.

\subsection{Prospect-refuge theory}

Incorporating Appleton's (1975) prospect-refuge theory, Fisher and Nasar (1992) created their general typology for evaluating an individual's perception of safety. They stated that an individual's level of prospect and the level of refuge for a potential offender (secondary refuge) are taken into consideration when people judge how safe they feel in an environment. Fisher and Nasar (1992) also cite Archea’s (1985) access-exposure model that highlights the role of visual access in perceptions of an environment to support their argument that the degree to which a space affords an opportunity to escape a potential attack plays a pivotal role in an individual's perception of safety. If little or an 
impeded escape is offered, the environment is less accessible and so an individual is more likely to perceive it as unsafe (more dangerous). It is possible that as a result of evolution, such environments tend to be least preferred while environments that offer early observation are associated with survival and tend to be preferred (Appleton, 1975). In support of this, research has demonstrated that people tend to prefer natural environments with open meadows surrounded by woods (e.g. Zube, Pitt \& Anderson, 1975). Woodland that receives the highest levels of preference tends to be deciduous, with little underbrush and an absence of grass cover (Daniel \& Bolster, 1976). In general, environments with less built elements, more distant views, topography change and clear water bodies receive higher preference ratings (Kaplan \& Kaplan, 1989; Steinitz, 1990; Zube et al., 1975).

Both field research and simulation techniques using Fisher and Nasar's (1992) typology have supported its claims. An environment that offers little prospect, contains a high level of refuge for a potential offender and offers an impeded escape for a potential victim is perceived as more dangerous and fear-evoking and is less preferred than an environment that offers prospect, contains a low level of refuge for a potential offender and offers a quick escape for a potential victim (Fisher \& Nasar, 1992; Nasar, Fisher \& Grannis, 1993; Nasar \& Jones, 1997; Petherick, 2000/2001; Wang \& Taylor, 2006). However these studies have only examined the typology within urban alleys and parklike spaces within university campuses. Although the examination of the typology within park-like spaces represents an exploration of people's experiences in natural environments, these are examples of natural spaces in urban areas and are often in close proximity to an area of high crime (e.g. Nasar et al., 1993; Nasar \& Jones, 1997). This 
study attempts to build on this body of research by exploring the typology within an exclusively natural environment.

There appears no reason as to why Fisher and Nasar's (1992) typology cannot be successfully applied to explain perceptions of danger or preference within an explicitly natural environment. There is a growing body of work within natural environments that has demonstrated that variables contributing to visibility and locomotor access are negatively related to perceived danger and fear but positively related to preference (e.g. Chapin, 1991; Herzog \& Kirk, 2005; Herzog \& Kropscott, 2004; Herzog \& Kutzli, 2002). Many of the features that contribute to visibility and locomotor access (e.g. curving pathways, vegetation, partial concealment and shadows, cf. Herzog \& Miller, 1998) are also regarded as features that enhance mystery. Herzog and Miller (1998) define mystery as the promise of further information from penetrating more deeply into a setting. However these features appear to have an extremely paradoxical role, having been shown to be a positive predictor of both preference (e.g. Herzog, 1987; Kaplan \& Kaplan, 1989) and perceived danger (e.g. Fisher \& Nasar, 1992; Nasar \& Fisher, 1993; Schroeder \& Anderson, 1984). Herzog and Bryce (2007) claim that in scenes of low prospect and visual access, mystery is often confused with surprise and so when properly understood, mystery is positively related to preference. As there is a high level of concordance with physical features that enhance mystery and preference with elements of Fisher and Nasar's (1992) typology, it appears that the typology could be successfully applied to natural environments.

This study attempts to examine the effect of the typology on perceived danger, fear and preference within a simulated example of a specific natural environment. A country 
park is a managed public open space environment within the United Kingdom that has a natural, rural atmosphere. Country parks are predominantly visited by those who do not want to venture into the wider countryside. In England there are over 270 designated country parks and they cover over 38,000 hectares of land. The majority of these country parks are managed and owned by local authorities, located on the rural-urban fringe and collectively receive an estimated 73 million visitors per year (The Countryside Agency, 2004).

\subsection{Perceptions of danger and fear}

Previous work has primarily measured perceived danger in terms of perceived likelihood ratings of coming to harm (e.g. Herzog \& Kirk, 2005; Herzog \& Kropscott, 2004; Herzog \& Kutzli, 2002). Although this is an important component of perceived danger, some researchers claim that perceived danger does not just consist of likelihood ratings, but also consequences of danger (Menzies \& Clarke, 1995; Williams, Turner \& Peer, 1985; Williams \& Watson, 1985) and the degree of control individuals feel they have over the situation (Rapee, 1997). The perceived danger measure used in this study will therefore incorporate likelihood ratings of coming to harm in addition to perceptions of severity and control.

This study will also examine how variations in the physical structure of the environment according to Fisher and Nasar's (1992) typology impact on fear ratings. Despite the strong positive correlations typically found between perceived danger and fear (e.g. Herzog \& Kutzli, 2002), there is a clear distinction between the two constructs. 
Perceived danger refers to a cognitive appraisal of danger while fear refers to an emotional reaction towards a stimulus or event. Gabriel and Greve (2003) claim that fear must always be accompanied by a cognitive element i.e. the cognitive perception of whether a situation is dangerous. However the distinction between emotional reactions such as fear and cognitive processes such as perceived danger is important. Although one expects a congruent emotional reaction in conjunction with a cognitive appraisal of danger, some sources of danger (e.g. extreme sports) may actually be perceived as attractive by some individuals, particularly if these dangers derive from nature. Van den Berg and ter Heijne (2005) found then when asked to describe a fearful experience with nature, participants recalled situations and events that evoked both negative and positive emotions. Overcoming the physical and psychological challenges of nature has also been shown to lead to a sense of enhanced well-being and self-esteem (Kaplan \& Kaplan, 1989).

\subsection{Dangers in natural environments}

Natural environments such as country parks contain a diverse range of potential dangers that very little previous research has tried to distinguish. Herzog and Smith's (1988) study using simulated urban alleys and narrow canyons distinguished between social and physical danger, with social danger defined as a danger stemming from a social source (e.g. being attacked by another person) while physical danger is defined as a danger stemming from the physical structure of the environment (e.g. being attacked by an animal, injury from tripping over obstacles, weather). The study found that only social 
danger was negatively related to preference. The threat of social danger in terms of physical or sexual assault in a natural environment is a very real one, particularly for women (Burgess, 1995; Coble, Selin \& Erickson 2003; Henderson \& Bialeschki, 1993). Other examples of potentially fear-evoking dangers that may be present in a natural environment include the weather, dangerous animals, the dark and falling branches (Bixler \& Floyd, 1997; van den Berg \& ter Heijne, 2005).

The effect of the physical structure of a natural environment may have on an individual's perception of how likely they could fall victim to these different types of dangers is something that does not appear to have been examined by previous research. Fisher and Nasar's (1992) typology is a useful framework for examining any potential differences in these perceptions because levels of prospect, refuge and escape are all likely to impact on perceptions of social and physical danger. A further potential danger that one could realistically encounter in a natural environment is becoming lost (Bixler \& Carlisle, 2004; Coble et al., 2003; Kaplan \& Talbot, 1983). There is some research which shows that variations in prospect, refuge and escape could be applied to the danger of becoming lost. Herzog and Kropscott (2004) demonstrated that both a lack of visual landmarks and a low level of movement ease acted as significant independent negative predictors of legibility, which in turn was a significant negative predictor of perceived danger. Therefore scenes low in legibility may prove disorientating and be associated with as harboring a greater likelihood of becoming lost.

As a result of the vast majority of existing research failing to distinguish between different types of danger in natural environments, very little is known about the relationships between different types of danger and more general constructs such as 
perceived danger, fear and preference. To date only the distinction between social and physical danger and their relationships with preference have been explored (Herzog \& Smith, 1988). With this study also measuring perceived danger, fear and preference as well as making a further distinction of the danger of becoming lost, the authors feel that exploring these relationships may reveal some interesting results that could contribute to the research area.

\subsection{Aim of study}

The study examines the effect of prospect-refuge on perceptions of danger, fear and preference. Three different simulated walks through a natural environment were developed with low, medium or high levels of prospect-refuge (Fisher and Nasar, 1992). From the reviewed literature, it is expected that a walk through a high prospect-low refuge environment (high levels of prospect and accessibility and few hiding places for a potential offender) will be perceived as less dangerous, fear-evoking and as harbouring a lower likelihood of encountering a social or physical danger or becoming lost than a walk through a similar environment with lower levels of prospect-refuge. Because of the higher adaptive value of an environment high in prospect-refuge to afford survival through greater forewarning over potential threats, it is also expected that respondents will prefer higher levels of prospect-refuge. This study also hopes to add to existing research by investigating the underlying relationships between the constructs, with perceived danger and fear both expected to have a negative relationship with preference. Given that the distinction between different types of danger is something that has 
received little attention by existing research, this study will also explore the relationships between the perceived likelihood of encountering specific types of danger and how they relate to perceived danger, fear and preference.

\section{Method}

\subsection{Respondents and design}

Two hundred and sixty nine respondents consisting of undergraduate, postgraduate and alumni members of a University in the South-East of England were recruited using a snowball sampling technique through the University's social networking intranet website (71 male, 198 female; $M=22.48$ years, $S D=7.84$ years; 18 - 27 years). The website contained a request for respondents on the home page, directing those interested in participating to click on a weblink to the online questionnaire immediately after finishing a working day they found fatiguing and stressful. Following the completion of the questionnaire, respondents were also asked to forward the link on to any other members of the networking site that they thought may be interested in participating in the study. Participation in the study was voluntary and no compensation was given.

Respondents were randomly assigned to one of three simulated environmental conditions that differed in levels of prospect-refuge according to Fisher and Nasar's (1992) typology (low, $n=90$; medium, $n=89$; high, $n=90$ ). This formed the single between-subject factor. 


\subsection{Environmental simulations}

The three environmental conditions were represented using a series of photographs that had been taken for this study in a country park in the south of England (The Queen Elizabeth Country Park near Portsmouth in the South of England). The park contains a diverse range of habitats from calcareous grassland and yew woodland to coniferous and beech plantations spread over more than 1400 acres that can be explored by walkers, cyclists and horse riders. An initial set of 124 photographs were taken, at a resolution of 2080 x 1544 pixels all on a summer afternoon under sunny and clear weather conditions. To prevent any attention being drawn away from the landscape, photographs did not contain other human beings or animals.

For the manipulation of prospect-refuge, a small pre-test was conducted with 7 respondents consisting of five students and two alumni ( 4 female; $M=32.15$ years, $S D=$ 8.16 years; $20-56$ years). Respondents were asked to place each of the 124 photographs into one of three piles representing low, medium or high agreement in response to three independent questions measuring prospect ('The extent your view is unobstructed to allow your field of vision to extend deep into the scene'), the number of hiding places ('The number of potential hiding places and opportunities for concealment') and accessibility ('The ease in which you can move through the scene'). This meant that respondents sorted all 124 photographs a total of three times. Photographs were shuffled between each sort and the order of the three questions was randomised between respondents to prevent order effects. 
Each photograph's mean rank for prospect, accessibility and number of hiding places was then created to allow the final slide selection to be made for each of the three environmental conditions. For the low prospect-high refuge condition, the photographs that were all ranked in both the bottom $20 \%$ for prospect and accessibility and the top $20 \%$ for hiding places were selected. For the medium prospect-medium refuge condition, the photographs that were all ranked in the middle $20 \%$ for prospect, accessibility and hiding places were selected. Finally for the high prospect-low refuge condition, the photographs that were all ranked in both the top $10 \%$ for prospect and accessibility and the bottom $20 \%$ for hiding places were selected. This process resulted in 36 photographs equally divided between the three conditions being selected for the use in the main study. To ensure the photographs selected were consistently classified into one of the three specific piles, the piles were coded (low agreement $=1$, medium agreement $=2$, high agreement $=3$ ) and a series of reliability tests using Crondbach's $\alpha$ were conducted. As can be seen in Table 1, the pilot test found a good level of internal consistency for the photographs selected to be used in the study.

Table 1. Range of reliability scores (Crondbach's $\alpha$ ) for the photographs selected for each prospect-refuge condition in response to prospect, hiding places and accessibility sort

The twelve photographs chosen for each condition were arranged into a believable sequence with respect to landscape and light to depict a short walk through the environment (see Figs. 1, 2 and 3). To help emphasise the physical structure of the 
environment, each condition was preceded by a short description of the physical structure and background to the walk that respondents were about to see in the photographs. Previous studies within the field have used descriptions to help respondents better imagine taking the walk for real (e.g. Staats, Gatersleben \& Hartig, 1997). Each description made indirect reference to levels of prospect, accessibility and hiding places by describing physical features such as light, vegetation, obstructions and pathways of the environment (see Appendix).

Fig. 1. Photograph from the low prospect-high refuge condition

Fig. 2. Photograph from the medium prospect-medium refuge condition

Fig. 3. Photograph from the high prospect-low refuge condition

\subsection{Measures}

Perceived danger was measured using 3 items that were phrased without reference to a specific source of danger: "How likely do you think it is that you could come to harm during your walk through this environment? How severe are the dangers you could potentially face walking through this environment? How well do you think you could control any potential dangers in this environment?" The response options once again ranged from 1 (not at all) to 7 (very much so) and permitted a scale score after responses from the third question were reversed (mean response) that ranged from 1 (not at all 
dangerous) to 7 (very dangerous). The scale had a respectable level of internal consistency (Crondbach's $\alpha=.73$ ).

Social danger as broadly defined by Herzog and Smith (1988) as 'danger that stems from a social source" was measured using 3 items: "How likely do you think it is that you could be followed by a stranger in this environment? How likely do you think it is that you could be assaulted by a stranger in this environment? How likely do you think it is that you could be mugged by a stranger in this environment?" The response options ranged from 1 (not at all) to 7 (very much so) that permitted a scale score (mean response) that ranged from 1 (very little social danger) to 7 (great deal of social danger). The scale had a high level of internal consistency (Crondbach's $\alpha=.94)$.

Physical danger as broadly defined by Herzog and Smith (1988) as 'danger that stems from the physical structure of the environment' was measured using 3 items: "How likely do you think it is that you could accidentally step on a snake in this environment? How likely do you think it is that you could become caught out in a violent thunderstorm? How likely do you think it is that you become injured by tripping over unseen obstacles?" The response options ranged from 1 (not at all) to 7 (very much so) that permitted a scale score (mean response) that ranged from 1 (very little physical danger) to 7 (great deal of physical danger). The scale had an acceptable level of internal consistency (Crondbach's $\alpha=.71)$.

Lost danger was measured using one item: "How likely do you think it is that you could lose your way and become lost walking through this environment?" The response options ranged from 1 (not at all) to 7 (very much so). 
Fear was measured using 3 items: "How frightened would you be taking a walk through this environment? How scared would you be taking a walk through this environment? How uneasy would you be taking a walk through this environment?" The response options ranged from 1 (not at all) to 7 (very much so) that permitted a scale score (mean response) that ranged from 1 (perceived as not at all fear evoking) to 7 (perceived as very fear evoking). The scale had a high level of internal consistency (Crondbach's $\alpha=.93$ ).

Preference was measured using 4 items that encompassed both beauty ratings and preference relative to other environments: "How pretty do you find this environment? To what extent do you like this environment? To what extent do you like this environment more than other natural environments you have visited? How beautiful do you find this environment?" The response options ranged from 1 (not at all) to 7 (very much so) and permitted a scale score (mean response) that ranged from 1 (not at all preferred) to 7 (very highly preferred). The scale had a high level of internal consistency (Crondbach's $\alpha$ $=.90)$.

One item was used to ensure there were no significant differences in previous experience of visiting country parks between the three experimental conditions: "How often do you visit country parks?" The response options ranged from 1 (not at all often) to 7 (very often). Also used to check the environmental manipulations, respondents were asked whether they felt the simulation was a representative a walk through a country park with the item: "Do you feel the photographs and description just shown to be a representative example of a walk through a typical country park in the United Kingdom?" The ratings ranged from 1 (not at all) to 7 (very much so). 


\subsection{Procedure}

An online questionnaire was developed for the study with respondents being e-mailed an online link to the study. The link instructed respondents to 'complete the questionnaire immediately following a working day that was stressful or fatiguing within a quiet environment without any distractions'. Following a brief explanation of the study, respondents were randomly assigned to one of the three environmental simulation conditions. They were given the short walk description and instructed to imagine they were taking the walk for real before viewing the twelve photographs. The photographs were presented as a slideshow. Each photograph was presented landscape at a size of $23.08 \mathrm{~cm} \times 17.13 \mathrm{~cm}$ and displayed for 3 seconds before merging into the next one using a fade-out technique for transition. The eight measures (perceived danger, social danger, physical danger, lost danger, fear, preference, previous environmental experience and accuracy of representation) were randomly split into two groups with respondents completing each of the three groups of measures in-between reading the description and seeing the slideshow. Following the completion of the eight measures, respondents were presented the slideshow one further time and asked to complete three manipulation check items that measured prospect, hiding places and accessibility. This resulted in the same simulated walk being shown a total of three times to respondents. This multiple exposure was done so that the walk remained salient for respondents when completing the measures rather than trying to complete all measures having only viewed the walk once. The order of both the items and the measures within each of the groups of measures was 
randomised to prevent order effects. After completing the measures, respondents were asked to record their age, gender and any further comments they may have had.

\subsection{Manipulation checks}

Although some researchers claim that differences in landscape preference ratings may be a function of age, gender and landscape exposure (e.g. Lyons, 1983) an extensive meta-analysis of landscape preference ratings by Stamps (1999) suggests a likely absence of individual differences related to similar demographics. Nonetheless from an experimental standpoint, it makes sense to have comparable groups. As can be seen in Table 2, no significant differences in age $\left(\chi^{2}(2)=0.22, p=.89\right)$, gender $\left(\chi^{2}(2)=0.22, p=.90\right)$ or experience of visiting country parks $(F(2,266)=2.48, p=.09)$ were found between the three environmental simulation conditions.

Table 2. Demographics (standard deviation) for the three prospect-refuge conditions

\section{On average respondents agreed that the simulated walk was largely representative of} a walk through a country park in the United $\operatorname{Kingdom}(M=5.24, S D=1.26)$. These ratings were not found to be overall significantly different between the three environmental simulation conditions $(F(2,266)=0.62, p=.54)$ while the planned contrasts in Table 3 failed to find any significant differences between individual environmental simulation conditions. 
Table 3. Mean representation example ratings (standard deviation) for the three prospectrefuge conditions

The same three questions from the pilot study were asked to check that the manipulation of prospect-refuge was successful ('The extent your view is unobstructed to allow your field of vision to extend deep into the scene'; 'the ease in which you can move through the scene'; 'the number of potential hiding places and opportunities for concealment'). The results revealed significant differences in ratings of prospect, accessibility and hiding places between the three experimental conditions (all $p$ 's<.001). Planned contrasts revealed the differences between each of the three conditions were significant and in the expected direction (see Table 4).

Table 4. Mean manipulation check ratings (standard deviation) for the three prospectrefuge conditions

\section{Results}

\subsection{Effect of prospect-refuge}

To test the effects of prospect-refuge, a series of one-way between-subjects analyses of variance (ANOVA) each with 3 planned contrasts (low vs. medium; low vs. high; medium vs. high) were conducted with prospect-refuge as the single between-subjects factor. The mean ratings for perceived danger, fear, types of danger and preference along 
with the results of the planned contrasts for each of the three prospect-refuge conditions are displayed in Table 5.

Table 5. Mean ratings (standard deviation) for the three prospect-refuge conditions

Significant differences in perceived danger $\left(F(2,266)=11.88, p<.001, \eta_{\mathrm{p}}{ }^{2}=.08\right)$ were found between the three prospect-refuge conditions. As can be seen in Table 5, the high prospect-low refuge environment was perceived as significantly less dangerous than both the medium $(t(266)=-2.16, p<.03, d=.26)$ and low prospect-high refuge environments $(t(266)=-4.87, p<.001, d=.60)$. The low prospect-high refuge environment was also perceived as significantly more dangerous than the medium prospect-medium refuge environment $(t(266)=2.69, p<.01, d=.33)$.

Similar findings were found in relation to fear, with significant differences found between the three prospect-refuge conditions following a Welch correction as the assumption of homogeneity had been broken $\left(F(2,175.13)=21.64, p<.001, \eta_{\mathrm{p}}{ }^{2}=.15\right)$. The high prospect-low refuge environment was evaluated as significantly less fearful than both the medium $(t(174.75)=-2.32, p<.03, d=.35)$ and low prospect-high refuge environments $(t(165.21)=-6.58, p<.001, d=.67)$. The low prospect-high refuge environment was also evaluated as significantly more fearful than the medium prospectmedium refuge environment $(t(172.02)=4.31, p<.001, d=.66)$. Although lower prospect and higherrefuge were found to be associated with Deleted: significantly higher ratings of perceived danger and fear, this was not found for perceptions of the perceived likelihood of encountering a social danger $(F(2,266)=$ 
1.82, $\left.p=.16, \eta_{\mathrm{p}}^{2}=.01\right)$. As can be seen in Table 5 , none of the contrasts between the three prospect-refuge conditions were significant. However significant differences were found between the three prospect-refuge conditions for the perceived likelihood of encountering a physical danger $\left(F(2,266)=5.25, p<.001, \eta_{\mathrm{p}}{ }^{2}=.04\right)$ and becoming lost $\left(F(2,266)=7.44, \mathrm{p}<.001, \eta_{\mathrm{p}}{ }^{2}=.05\right)$. Planned contrasts revealed a significantly greater perceived likelihood of encountering a physical danger in the low prospect-high refuge environment as opposed to the medium $(t(266)=1.82, p<.05, d=.22)$ and the high prospect-low refuge environments $(t(266)=-3.23, p<.001, d=.40)$. However the chance of encountering a physical danger was perceived as equally likely between the medium and high prospect-low refuge environments $(t(266)=1.40, p=.08, d=.17)$. In terms of the perceived likelihood of becoming lost, planned contrasts revealed that this was significantly lower in the high prospect-low refuge environment than in both the low $(t$ $(266)=-3.82, p<.001, d=.47)$ and medium prospect-medium refuge environments $(t$ $(266)=-2.39, p<.01, d=.29)$. However the perceived likelihood of becoming lost was judged equally likely between the low and medium prospect-medium refuge environments $(t(266)=1.42, p=.08, d=.17)$.

Significant differences in preference were also found between the three prospectrefuge conditions $\left(F(2,266)=13.77, p<.001, \eta_{\mathrm{p}}{ }^{2}=.09\right)$. As can be seen in Table 5, although the high prospect-low refuge environment was preferred over the low prospecthigh refuge environment $(t(266)=5.03, p<.001, d=.62)$, it was not preferred significantly more than the medium prospect-medium refuge environment $(t(266)=1.22$, $p=.22, d=.15)$. However the medium prospect-medium refuge environment was 
preferred over the low prospect-high refuge environment $(t(266)=3.80, p<.001, d=$ $.47)$.

\subsection{Relationships between variables}

To investigate the relationships between variables, a series of linear and multiple regressions were conducted. A moderate negative relationship (Spearman's $\rho=-.32$, $p<.001)$ was found between perceived danger and preference $\left(R^{2}{ }_{\text {adj }}=10.1 \%, F(1,267)=\right.$ $31.27, p<.001)$. A stronger negative relationship between fear and preference was found (Spearman's $\rho=-.51, p<.001$ ) with the regression model once again revealing a significant fit $\left(R^{2}{ }_{\text {adj }}=25.4 \%, F(1,267)=92.07, p<.001\right)$. Further analysis using Fisher's Z-transformation revealed fear to be a significantly stronger negative predictor of preference than perceived danger $(Z=-6.37, p<.001)$.

Perceived likelihood ratings of encountering the three different types of danger were also used as predictor variables in a multiple regression examining the effect on preference. The three ratings of the dangers were found to share a significant but moderate negative relationship with preference (Spearman's $\rho=-.38, p<.001$ ) and the overall regression model revealed a significant fit $\left(R^{2}\right.$ adj $=13.6 \%, F(3,265)=15.05$, $p<.001)$. However as can be seen in Table 6 , only the perceived likelihood of encountering social and lost dangers were found to have a significant negative effect on preference. 
Table 6. Summary of multiple regression statistics for the predictor variables on preference ratings

Perceived likelihood ratings of encountering the three different types of dangers were also found to share a significant and strong positive relationship with perceived danger (Spearman's $\rho=.70, p<.001)$. Once again the overall regression model produced a significant fit $\left(R^{2}{ }_{\text {adj }}=47.8 \%, F(3,265)=82.93, p<.001\right)$ with all three perceived likelihood danger ratings having a significant positive effect on perceived danger (see Table 7).

Table 7. Summary of multiple regression statistics for the predictor variables on perceived danger ratings

The three perceived likelihood ratings of the dangers were also found to share a significant and strong positive relationship with fear (Spearman's $\rho=.59, p<.001)$. The overall regression model once again produced a significant fit $\left(R^{2}{ }_{\text {adj }}=34.3 \%, F(3,265)\right.$ $=47.73, p<.001)$. As can be seen in Table 8 , only the perceived likelihood of encountering a social danger or becoming lost had a significant positive effect on fear.

Table 8. Summary of multiple regression statistics for the predictor variables on fear ratings

\section{Discussion}


This study attempted to examine how variations in the physical structure of a simulated natural environment influenced perceptions of danger, fear and preference before exploring the relationships between these variables.

\subsection{The effect of prospect-refuge}

Using Fisher and Nasar's (1992) typology as a framework, significant expected differences in perceived danger, fear and preference were found between the simulated walks through the three prospect-refuge conditions. Low prospect-and high refuge was, perceived as more dangerous and fearful but less preferred than high prospect and low refuge These results are consistent with Fisher and Nasar's (1992) typology and emphasise the roles of prospect, refuge and escape on perceptions made within an environment. Existing work using the typology has been largely confined to urban alleyways and park-like spaces within university campuses (e.g. Fisher \& Nasar, 1992; Fisher, Nasar \& Grannis, 1993; Nasar \& Jones, 1997; Petherick, 2000/2001; Wang \& Taylor, 2006). The results of this study suggest that the typology can also be successfully applied beyond urban environments and natural spaces within urban areas to a distinct natural environment to explain variations in perceptions of danger, fear and preference.

This study also examined how variations in the physical structure of the environment according to the typology impacted on perceptions of the likelihood of one encountering three specific types of danger. The results suggested that variations in prospect-refuge levels had a significant impact on perceptions of the likelihood of encountering both a 
physical danger and becoming lost. The direction of these results was as expected, with low prospect and high-refuge perceived as harbouring a greater chance of falling victim to these dangers. However no significant differences were found for the perception of how likely one could encounter a social danger. At face value, this result is highly surprising. Fisher and Nasar's (1992) typology of prospect-refuge is based upon the risk of social danger, an individual's perception of safety from a potential offender within an urban environment. Existing work within urban environments has found low_prospect and high refuge is associated with higher perceptions of becoming a victim of social danger

Deleted: er levels of Deleted: Deleted: to be (e.g. Petherick 2000/2001; Wang \& Taylor, 2006). Even work within urban parks has demonstrated high levels of dense understory vegetation that impedes prospect and offers potential attackers a place to hide to be associated with a higher fear of crime (e.g. Fisher \& Nasar, 1992; Kuo, Bacaicou \& Sullivan, 1998; Troy \& Grove, 2008). Indeed Micheal, Hull and Zahm (2001) claim that vegetation that impedes prospect is likely to facilitate criminal activity and this is likely to result in not just an increased fear of crime, but also an increased perceived likelihood of crime. However it is worth considering that the majority of these existing studies have been conducted in North America whereas this study has been conducted within a specific example of a natural environment within the United Kingdom. Therefore it is possible that the contrasting results of this study could be attributed to cultural differences. However as previously stated, country parks tend to be much larger and provide a far more natural rural atmosphere than urban parks and so the authors feel the contrasting results are a result of environmental differences. For example, people may expect country parks to contain far less of the criminal and social dangers that urban environments have become associated with. Therefore people may 
perceive the perceived likelihood of encountering a social danger as being much lower in a country park environment than in an urban park or urban environment. Indeed mean ratings for the likelihood of encountering a social danger fell midway on the scale suggesting that people only perceived a moderate likelihood of a encountering a social danger. Because a country park is not typically associated with social danger, prospectrefuge may not actually be that important and respondents may have generalised the perceived likelihood of encountering a social danger in terms of a country park regardless of its physical structure.

Kuo and Sullivan (2001) report that in North America, active programs to remove vegetation in urban parks are being followed in an attempt to reduce the perceived likelihood and fear of crime. Indeed Forsyth and Musacchio (2005) dedicate an entire chapter in their book 'Designing Small Parks' to improving perceptions of safety from crime and suggest the removal of vegetation that offers concealment because people tend to feel safer when the understory is clear. Other guidelines for the design of urban parks include good lighting and clear view corridors (Forsyth \& Musacchio, 2005). Whilst these design guidelines may work for urban parks, the results of this study suggest that it is unlikely that changes to improve prospect and remove refuge for a potential offender will have a significant impact on reducing people's perceptions of social danger within a country park. It should also be noted that perceptions may vary during different times of day. For example, Fisher and Nasar (1992) have demonstrated that fear intensifies after dark and so the perception of a fear-evoking threat or danger is more likely to have a larger detrimental effect on preference in the evening than it does during the day. Although people are unlikely to visit natural environments such as country parks at night, 
they may still be visitors at times when it starts to get dark. At times such as these, the perception of a fear-evoking danger may have a greater impact than at brighter times of the day.

Of course it seems plausible that the physical structure of the environment is more likely to play some part in an individual's perceptions and emotional reactions if a social danger is actually present. The researchers are currently examining whether variations in prospect, refuge and escape consistent with the typology can evoke significant differences in perceptions of danger, fear and preference if an implied social danger is encountered during a simulated walk through a country park.

\subsection{The relationships between perceived danger, fear, specific types of danger and preference}

The distinction between perceived danger and fear was highlighted earlier in this study and appears to be warranted because although both constructs were significant negative predictors of preference, fear was a significantly stronger negative predictor of preference than perceived danger. One possible explanation for this is that perceptions of danger can sometimes be attractive e.g. extreme sports. Conversely fear represents an extremely unpleasant emotional reaction coupled with heightened arousal that has become associated with a threat to human survival. This means that fear is far less likely to be associated with positive cognitions or emotions than perceptions of danger. Fear can even lead to people avoiding places that become associated with it (Keane, 1998). 
Although fear normally accompanies perceptions of danger, this may not always be the case. The results demonstrated that perceptions of physical danger were related to general perceptions of danger but not to fear. Fear and preference in this study were only related to perceptions of social and physical danger and not to physical dangers. This may be because social dangers and the danger of getting lost were more strongly associated with threats to survival than physical dangers. Many incidents of physical danger are not so severe as to threaten survival which would be likely to evoke fear. For example, physical challenges such as climbing over tricky obstacles, crossing a stream and making one's way through brambles could cause injury and be perceived as dangerous, but they are unlikely to evoke fear because they represent rather minor dangers that do not threaten survival. Indeed successfully confronting the physical challenges and dangers that 'lurk in the woods' may lead to individuals feeling refreshed and invigorated (Kaplan $\&$ Talbot, 1983). Existing research has found that when coming into contact with sources of physical danger in a natural environment, people may experience negative as well as positive emotions (e.g. Kaplan \& Talbot, 1983; van den Berg \& ter Heijne, 2005). These positive emotions may dilute negative emotions such as fear and may help explain why unlike the other types of dangers, the perceived likelihood of encountering a physical danger was not found to have a significant effect on fear.

These results clearly support the distinction between different types of danger being made which is something that has received little attention in existing research. However only a small sample of the physical dangers that may be present in a natural environment were explored in this study. Future research in the area may like to disseminate specific types of danger further. For example, examining both animate (e.g. animals, snakes etc.) 
and inanimate sources of physical danger (e.g. obstacles and physical features that could lead to personal injury) may warrant future exploration as the threat they pose to survival and their subsequent effects on perceptions of an environment may differ.

\subsection{Limitations}

Several methodological issues regarding this study warrant consideration. Having respondents rate both dependent measures (types of danger, perceived danger, fear and preference) alongside the independent measures of prospect, hiding places and accessibility may have given risen to a bias whereby completing one set of measures influences the other. Because of this, relationships found in the regression analyses may be overstated. However this will not have affected the results of the analyses of variance as respondents completed the ratings of danger, fear and preference before the ratings of prospect and refuge. This means that there was no opportunity for the independent measures to influence the dependent measures. Although there remained an opportunity for the completion of the dependent measures to influence the independent measures, the strong congruence in ratings of the independent measures with the pilot study (with a different group of respondents) can allay this concern. Moreover although the strength of the relationships in the analyses of variance may have been affected, there is no reason to assume that the pattern of relationships may have been aversely affected. Therefore although the regression findings should be interpreted with care, they do still provide significant insight, into the previously unexplored relationships between environmental design, perceptions of danger, fear and preference. 
It should also be acknowledged that the use of an online questionnaire does give rise to potential variations between respondents, including differences between computer specifications and sizes that may have resulted in significant variations in the size and quality of the images respondents saw. However the photographs were presented at a specific resolution and size that the vast majority of modern laptop and desktop computers could project. There is also no reason to believe that such random error was systematically different between experimental groups. An additional concern that should be noted when collecting data via an on-line procedure is a lack of control over the sampling procedure. Further research may therefore, need to verify the current findings using different sampling strategies. In this present study, however, every attempt was made to alleviate any problems resulting from this procedure. Although we have no reason to believe that respondents did not follow the instructions regarding completing the questionnaire at the end of a fatiguing day, we cannot be completely sure they did. We felt that asking respondents to complete the questionnaire at the end of a fatiguing day represented a more ecologically valid technique than making respondents complete the questionnaire at a specified time when they may not be fatigued. Staats, Kievet and Hartig (2003) demonstrated a heightened preference for a natural environment when in a state of attentional fatigue. If, as one would expect, an increased preference for an environment means an individual is more likely to visit it, then ensuring respondents are in a state where they are more likely to actually visit the environment improves the ecological validity of our methodology.

The use of a simulation method invariably evokes reservations regarding ecological validity. Despite the walks being created using disparate photographs from the same 
environment, responses from a manipulation check item indicated that the descriptions and photographs were considered as representative of walks through a country park. Nonetheless, despite sequential slides being successful in providing route information, they are not successful for providing spatial information (Hunt, 1993). Spatial information is likely to be very different for scenes of low and high prospect-low refuge, with low prospect-high refuge scenes characterised by enclosed surroundings that make much it harder to navigate and pinpoint one's location than high prospect-low refuge scenes. However the manipulation check did suggest that anticipations of getting lost and losing orientation were significantly different between conditions in the expected direction. These results suggest that the method employed was successful in getting respondents to generate some level of spatial-orientation information.

The simulation technique used also restricts respondents to only experience the visual characteristics of a walk through a country park environment. Other characteristics that may be considered integral to the experience such as smell and touch were not experienced by the respondents. Being asked to imagine making the walk is also essentially a passive experience, not allowing the behavioural freedom and decision making that invariably forms part of a real walk. Environmental simulation has a relatively long history in environmental psychology and environmental assessment judgments (cf. Marians \& Stokols, 1993). However the use of simulations can only make it harder to detect any real effects and should therefore be supported with field experiments.

The use of a student or recently graduated sample also invariably raises questions about the generalisability of the results to other demographics and groups. A meta- 
analysis by Stamps (1999) provides grounds for confidence that such a sample may be generalisable to the general population, but future research is needed to settle the debate.

\subsection{Implications}

Within the United Kingdom a number of public health strategies have been developed including the 'Active Woods - naturally good for you' campaign which encourages participation in outdoor recreation and the exploration of natural environments such as country parks. These environments represent an important tool to help improve the physiological and psychological well-being of an increasingly urbanized population within the United Kingdom. The success of these strategies will ultimately rely on visitors keen to explore these environments and so it is essential that they are perceived as preferable and safe environments that facilitate exploration, interaction and discovery.

Expected differences in overall ratings of perceived danger, fear and preference between the three conditions further highlight the importance of physical features such as prospect, refuge and potential escape in how an individual perceives and experiences a natural environment. The manipulation of these physical features so that there is a high level of prospect, a low level of refuge and an unobstructed potential escape route appears one way of reducing perceptions of danger and fear while making the environment more preferable. This is likely to be due in part to such a physical structure reducing the perceived likelihood of encountering a physical danger or becoming lost. However the physical structure of the environment does not seem to influence the perceived likelihood of encountering a social danger. Given that this type of danger appears to be highly fear- 
evoking and damaging to preference, other ways of managing the perception of social danger needs to be explored to help protect the value of natural environments.

\section{References}

Appleton, J. (1975). The experience of place. London: Wiley.

Archea, J.C. (1985). The use of architectural props in the conduct of criminal acts. The Journal of Architectural and Planning Research, 2, 245-259.

Bixler, R. D. \& Carlisle, C. L. (1994). Observed fears and discomforts among urban students on field trips to wildland areas. Journal of Environmental Education, 26, 24-33.

Bixler, R. D. \& Floyd, M. F. (1997). Nature is scary, disgusting and uncomfortable. Environment and Behavior, 29, 443-467.

Blöbaum, A. \& Hunecke, M. (2005). Perceived danger in urban public space: The impacts of physical features and personal factors. Environment and Behavior, 37, 465486.

Burgess, J. (1995). Growing in confidence - understanding people's perceptions of urban fringe woodlands. Cheltenham, United Kingdom: Countryside commission. 
Chapin, D. (1991). Making green places safer places: Experiences in New York City. Landscape Architecture Review, 12, 16-18.

Coble, T. G., Selin, S. W. \& Erickson, B. B. (2003). Hiking alone: Understanding fear, negotiation strategies and leisure experience. Journal of Leisure Research, 35, 1-22.

Daniel, T. C. \& Boster, R. S. (1976). Measuring landscapes aesthetics: The SBE method. Boulder: USDA.

Fisher, B. S. \& Nasar, J. L. (1992). Fear of crime in relation to three exterior site features: Prospect, refuge, and escape. Environment and Behavior, 24, 35-65.

Forsyth, A. \& Musacchio, L. (2005). Designing small parks: A manual for addressing social and ecological concerns. Hoboken, NJ: John Wiley \& Sons.

Gabriel, U. \& Greve, W. (2003). The psychology of fear of crime. Conceptual and methodological perspectives. The British Journal of Criminology, 43, 600-614.

Henderson, K. A. \& Bialeschki, M. D. (1993). Fear as a constraint to active lifestyles for females. Journal of Physical Education, Recreation and Dance, 44-47.

Herzog, T. R. (1987). A cognitive analysis of preference for natural environments: mountains, canyons, deserts. Landscape Journal, 6, 140-152. 
Herzog, T. R. \& Bryce, A. G. (2007). Mystery and preference in within-forest settings. Environment and Behavior, 39, 779-796.

Herzog, T. R. \& Flynn-Smith, J. A. (2001). Preference and perceived danger as a function of the perceived curvature, length and width of urban alleys. Environment and Behavior, $33,655-668$

Herzog, T. R. \& Kirk, K. M. (2005). Pathway curvature and border visibility as predictors of preference and danger in forest settings. Environment and Behavior, 37, 620-639.

Herzog, T. R. \& Kropscott, L. S. (2004). Legibility, mystery, and visual access as predictors of preference and perceived danger in forest settings without pathways. Environment and Behavior, 36, 659-677.

Herzog, T. R. \& Kutzli, G. E. (2002). Preference and perceived danger in field/forest settings. Environment and Behavior, 34, 819-835.

Herzog, T. R. \& Miller, E. J. (1998). The role of mystery in percieved danger and environmental preference. Environment and Behavior, 30, 429-449.

Herzog, T. R. \& Smith, G. A. (1988). Danger, mystery and environmental preference. Environment and Behavior, 20, 320-344. 
Hunt, M. E. (1993). Environmental simulation research for an aging society.

Familiarizing older people with the unknown. In R. W. Marans \& D. Stokols (Eds.),

Environmental Simulation: Research and Policy (pp. 87-112.). New York: Plenum Press.

Kaplan, R. \& Kaplan, S. (1989). The experience of nature: A psychological perspective.

Cambridge: Cambridge University Press.

Kaplan, S. \& Talbot, J. F. (1983). Psychological benefits of a wilderness experience. In I. Altman \& J. Wohlwill (Eds.), Human behavior and environment: Advances in theory and research, Vol. 6 (pp. 163-203). New York: Plenum Press.

Kuo, F. E., Bacaicoa, M. \& Sullivan, W. C. (1998). Transforming inner-city landscapes. Environment and Behavior, 30, $28-59$.

Kuo, F. E. \& Sullivan, W. C. (2001). Environment and crime in the inner city.

Environment and Behavior, 33, 343-367.

Lyons, E. (1983). Demographic correlates of landscape preference. Environment and Behavior, 15, 487-511.

Marans, R. W. \& Stokols, D. (1993). Environmental simulation: Research and policy. New York: Plenum Press. 
Menzies, R. G. \& Clarke, J. C. (1995). Danger expectancies and insight in acrophobia. Behaviour Research and Therapy, 33, 215-221.

Michael, S., Hull, R. \& Zahm, D. (2001). Environmental factors influencing auto Burglary - a case study. Environment and Behavior, 33, 368-388.

Nasar, J. \& Fisher, B. (1993). Hot spots' of fear and crime: a multi-method investigation. Journal of Environmental Psychology, 13, 187-206.

Nasar, J., Fisher, B. \& Grannis, M. (1993). Priximate cues to fear of crime. Landscape and Urban Planning, 26, 161-178.

Nasar, J. \& Jones, K. (1997). Landscapes of fear and stress. Environment and Behavior, 29, 291-323.

Petherick, N. (2000/2001). Environmental design and fear: The prospect-refuge model and the university college of the Cariboo campus. Western Geography, 10/11, 89-112.

Rapee, R. (1997). Perceived threat and perceived control as predictors of the degree of fear in physical and social situations. Journal of Anxiety Disorders, 11, 455-461.

Schroeder, H. W. \& Anderson, L. M. (1984). Perceptions of personal safety in urban recreation sites. Journal of Leisure Research, 16, 178-194. 
Staats, H., Gatersleben, B. \& Hartig, T. (1997). Change in mood as a function of environmental design: Arousal and pleasure on a simulated forest hike. Journal of Environmental Psychology, 17, 288-300.

Staats, H., Kievet, A. \& Hartig, T. (2003). Where to recover from attentional fatigue: An expectancy-value analysis of environmental preference. Journal of Environmental Psychology, 23, 147-157.

Stamps, A., E. (1999). Demographic effects in environmental aesthetics: A meta-analysis. Journal of Planning Literature, 14, 155-175.

Steinitz, C. (1990). Toward a sustainable landscape with high visual preference and high ecological integrity: The loop road in Acadia national park USA. Landscape and Urban Planning, 19, 213-250.

The Countryside Agency (2004). Towards a country park renaissance. Available at: www.countryside.gov.uk/WiderWelcome/Countryside_Recreation/countryParks/index.as $\mathrm{p}$

Troy, A. \& Grove, J. M. (2008). Property values, parks, and crime: A hedonic analysis in Baltimore, MD. Landscape and Urban Planning, 87, 233-245. 
Van den Berg, A. E. \& ter Heijne, M. (2005). Fear versus fascination: An exploration of emotional responses to natural threats. Journal of Environmental Psychology, 25, 261272.

Wang, K. \& Taylor, R. B. (2006). Simulated walks through dangerous alleys: Impacts of features and progress on fear. Journal of Environmental Psychology, 26, 269-283.

Williams, S. L., Turner, S. M. \& Peer, D. F. (1985). Guided mastery and performance desensitization treatments for severe acrophobia. Journal of Consulting and Clinical Psychology, 53, 237-247.

Williams, S. L. \& Watson, N. (1985). Perceived danger and perceived self-efficacy as cognitive determinants of acrophobic behavior. Behavioral Therapy, 16. 136-146.

Wilson, E. O. (1984). Biophilia. Cambridge: Harvard University Press.

Zube, E. H., Pitt, D. G. \& Anderson, T. W. (1975). Perception and prediction of scenic re-sources values of the Northeast. In Zube, E. H., Brush, R. \& Fabos, J. (Eds.) Landscape assessment: Values, perceptions and resources (pp. 151-167). New York: Dowden, Hutchinson and Ross.

\section{Appendix - walk descriptions for the three prospect-refuge conditions}


